\title{
Procalcitonin Elevation without Sepsis: Can it be a Tumor Marker? A Case Report and Literature Review
}

\section{Keskin 0* \\ Department of Internal Medicine, Selcuk University Medical Faculty, Turkey}

*Corresponding author: Ozge Keskin, Department of Internal Medicine, Selcuk University Medical Faculty, Turkey, Tel: 5054031968; Email: odurdu2000@yahoo.com

\section{Mini Review}

Volume 3 Issue 2

Received Date: July 16, 2019

Published Date: July 25, 2019

DOI: $10.23880 /$ hij-16000141

\section{Abstract}

Procalcitonin is a protein which has an important role in diagnosis and predicting prognosis of sepsis. Despite sepsis procalcitonin levels were found to be high in some malignant patients especially in neuroendocrine tumors. There are studies showing the relationship between procalcitonin levels and disease stage, prognosis and recurrence. Most of the cases in these studies are medullary thyroid cancer cases. There was limited data about procalsitonin level in other solid tumors. Our case is a metastatic lung cancer patient with neuroendocrine component.

Keywords: Procalcitonin; Neuroendocrine; Lung cancer; Sepsis

Abbreviations: CRP: C - reactive protein; CT: Computer Tomography.

\section{Introduction}

Calcitonin is a part of a protein which is a product of CALC1 gene. It is a polypeptide hormone composed of 32 amino acids. Procalcitonin is the precursor peptide of calcitonin and it consists of 116 amino acids and weighs $13 \mathrm{kDa}$ [1]. Procalcitonin is more heat-resistant than calcitonin and changes in the circadian level are less common. Calcitonin is more unstable at room temperature and therefore may have false-negative results [2]. Neuroendocrine tumors develop from enterochromaffin cells in neuroendocrine tissue. These cells are located in the intestinal system, pancreas and lung [1]. These neuroendocrine cells secrete procalcitonin. Among these, the most important procalcitonin factory is thyroid C cells. Herein we reviewed the literature because of a metastatic lung neuroendocrine carcinoma patient without sepsis, in which high procalcitonin level regressed after chemotherapy treatment.

\section{Case Report}

A 63-year-old man presented with abdominal pain and constipation. The patient had no known chronic disease and no medication. In physical examination; auscultation of the right lung was pathologic. Abdominal examination revealed tenderness in the right upper quadrant. The liver was palpable $4 \mathrm{~cm}$ below the rib. Laboratory findings revealed significantly high procalcitonin level, except anemia and elevated liver function tests. Simultaneous procalcitonin and C-reactive protein (CRP) levels were $31.2 \mu \mathrm{g} / \mathrm{L}(<0.5 \mu \mathrm{g} / \mathrm{L}$ normal $)$ and $10.1 \mathrm{mg} / \mathrm{L}$ ( $<8 \mathrm{mg} /$ $\mathrm{L}$ normal), respectively. The patient was afebril during these tests. Additional laboratory tests and cultures were 
obtained for infection and the patient was started on empirical antibiotics. Thorax computed tomography (CT) revealed two masses of $13 \times 25 \mathrm{~mm}$ and $25 \times 31 \mathrm{~mm}$ in the upper lobe of the right lung. Abdominal CT showed multiple metastatic lesions in the liver and a mass with a diameter of $30 \mathrm{~mm}$ in the rectum. There were multiple metastatic bone lesions on both tomography scans. During the test period, the liver biopsy performed prior to hospitalization resulted. Immunohistochemical findings supporting large cell neuroendocrine carcinoma of the lung were detected. These were; Synaptophysin, CD56, Villin positivity, TTF-1 focal positivity, Napsin-A, MUC5AC negativity. During antibiotic treatment, CRP level decreased to $4.84 \mathrm{mg} / \mathrm{L}$ while there was no change in procalcitonin level $(30.4 \mu \mathrm{g} / \mathrm{L})$. The patient underwent cisplatin based chemotherapy protocol for malignancy. The procalcitonin value decreased to $21.8 \mu \mathrm{g}$ / L, two days after the completion of chemotherapy. Informed consent was obtained from the patient for this case report.

\section{Discussion}

Procalcitonin is produced in response to inflammation. Therefore, it has an important role in diagnosis and predicting prognosis of sepsis. But also, calcitonin and procalcitonin levels were found to be high in some malignant patients. These malignant diseases are neuroendocrine tumors of various organs, especially thyroid medullary carcinoma and small cell lung cancer. In some hematologic tumors, procalcitonin level was found to be elevated without an infection [1-3]. Cate were the first to mention the paraneoplastic production of calcitonin and hence procalcitonin in neoplatic diseases. In subsequent studies, calcitonin and procalcitonin levels were also correlated with disease stage [2].

Karagiannis, et al. rewieved medullary thyroid cancer cases. Procalcitonin level varies according to disease stage and lymph node involvement in these cases. Procalcitonin levels are higher in recurrent and /or advanced disease [2]. Cases in this review were mostly with medullary thyroid cancer, differentiated thyroid cancers and benign thyroid diseases were also included. Procalcitonin level was higher in malignant diseases and tumor stage was associated with procalcitonin level. Similar to case reports, there was a relationship between tumor size, lymph node involvement and disease stage and procalcitonin level in the clinical studies. Procalcitonin levels decreased to normal in the postoperative period when there is no residual thyroid tissue. In addition, the ratio of procalcitonin to calcitonin was found to be prognostic in some studies. High procalsitonin/calsitonin ratio after treatment (this ratio is defined as 2.4 in the study) is a negative prognostic factor [2].

Unlike the previous review, neuroendocrine tumors other than medullary thyroid cancer are also included in the review of Qida $\mathrm{H}$, et al. These include; lung, intestine and pancreatic neuroendocrine tumors [1,4]. Some studies in this review also found a relationship between disease stage and procalcitonin level. Procalcitonin level is higher in metastatic disease. Elevated procalcitonin levels in non-thyroid solid organ tumors are due to intratumor neuroendocrine component. As the grade of this neuroendocrine component increases, the procalcitonin level also increases [1].

In the study of Avrillon, et al. lung cancer cases were examined. Procalcitonin levels were significantly higher in tumors with neuroendocrine component and with metastatic disease. Procalcitonin levels significantly increased in patients with liver metastases and as the number of metastatic lesions increased [3]. Similar results were found in the study of Patout, et al. Procalcitonin levels are higher in lung cancer patients with neuroendocrine component and in patients with metastatic disease. In this study, high procalcitonin level was found to be a negative prognostic factor [5].

Despite all these data, the use of procalcitonin is still limited for the diagnosis and follow-up of malignant disease. However, there is a light that it can be a guide especially in neuroendocrine tumors.

\section{References}

1. Hu Q, Jin P, Zhao X, Wu W, Huang B, et al. (2018) Esophageal neuroendocrine carcinoma complicated with unexpected hyperprocalcitonin: Case report and literature review. Medicine 97(40): 12219.

2. Karagiannis AK, Girio-Fragkoulakis C, Nakouti T (2016) Procalcitonin: A New Biomarker for Medullary Thyroid Cancer? A Systematic Review. Anticancer Res 36(8): 3803-3810.

3. Avrillon V, Locatelli-Sanchez M, Folliet L, Carbonnaux M, Perino E, et al. (2015) Lung cancer may increase serum procalcitonin level. Infect Disord Drug Targets 15(1): 57-63.

4. Takahashi K, Ozawa E, Nakao K, Aoki S, Takase Y (2019) Hepatobiliary and Pancreatic: A procalcitoninsecreting and calcitonin-secreting pancreatic 


\section{Haematology International Journal}

neuroendocrine carcinoma. J Gastroenterol Hepatol 34(6): 964.

5. Patout M, Salaün M, Brunel V, Bota S, Cauliez B, et al. (2014) Diagnostic and prognostic value of serum procalcitonin concentrations in primary lung cancers. Clin Biochem 47(18): 263-267. 